\title{
3-fach-Prophylaxe auch für Menschen in der zweiten Lebenshälfte
}

\begin{abstract}
Die Anzahl der jüngeren Senioren', die einen guten Mundgesundheitsstatus angibt, konnte sich in den letzten 20 Jahren fast verdreifachen - eine positive Tendenz. ${ }^{2}$ Neben regelmäßigen zahnmedizinischen Vorsorgeuntersuchungen ist gerade auch die tägliche häusliche Zahnpflege mit geeigneten prophylaktischen Hilfsmitteln für den langfristigen Erhalt der Mundgesundheit essentiell. Mundspülungen mit ätherischen Ölen (z.B. Listerine ${ }^{\oplus}$ ) bieten im Rahmen der täglichen 3-fach-Prophylaxe eine wertvolle Ergänzung der mechanischen Zahnreinigung. Sie erreichen auch schwer zugängliche Bereiche des Mundraums, die bei der mechanischen Zahnreinigung unberücksichtigt bleiben können. ${ }^{3}$
\end{abstract}

Die Ergebnisse der Fünften Deutschen Mundgesundheitsstudie (DMS V) zeigen eine deutliche Verbesserung in der zahnmedizinischen Gesundheitsversorgung. So verschieben sich die Krankheitslasten immer mehr in das höhere Lebensalter. Das führt zu deutlich mehr mundgesunden Lebensjahren: Nur noch jeder achte jüngere Senior ist zahnlos, im Jahr 1997 war es noch jeder vierte.' Demnach betreiben viele Senioren heutzutage eine bessere Mundhygiene. ${ }^{4}$ Dennoch bleiben Zahn- und Zahnfleischprobleme im Alter nicht aus.

"Prophylaxe beim Zahnarzt: Orale Gesundheit im Alter Mit den Jahren nutzen sich Zähne durch Beißen, Kauen, Reißen und Schleifen sowie durch regelmäßige Angriffe säurehaltiger Speisen und Getränke ab. Prophylaxe ist deshalb in jeder Lebensphase unverzichtbar. Im Rahmen eines umfassenden Prophylaxekonzepts in der Zahnarztpraxis sollten im Vorfeld Faktoren ermittelt werden, die bei der Entstehung oder Entfernung des dentalen Biofilms eine Rolle spielen: dazu zählen Ernährung, Mundhygieneverhalten, Zahnstellung und Restaurationen. ${ }^{5}$ Gerade die Behandlung von älteren Patienten mit Vorerkrankungen und gesundheitlichen Risikofaktoren kann das zahnärztliche Fachpersonal vor besondere Herausforderungen stellen. Um unerwünschte Neben- und Wechselwirkungen einer Behandlung auszuschließen, sollte im Vorfeld ein ausführliches Anamnesegespräch stattfinden.

\section{"Verbesserung der häuslichen Mundpflege durch tägliche 3-fach-Prophylaxe}

Basis für den langfristigen Erhalt der Zahn- und Mundgesundheit ist ein gutes Biofilmmanagement. Eine effektive Biofilmkontrolle setzt bei der täglichen mechanischen Zahn- und Interdentalreinigung an. Dennoch ist die mechanische Zahnreinigung allein nicht immer ausreichend, um den Biofilm effektiv zu entfernen. Da Zähne mit ca. $25 \%$ nur einen kleinen Anteil des Mundraums ausmachen, befreit die mechanische Reinigung meist nicht den gesamten Mundraum vom Biofilm. Um auch diese Reste des dentalen Biofilms effektiv zu bekämpfen, ist die ergänzende tägliche Anwendung einer antibakteriellen Mundspülung im Rahmen der

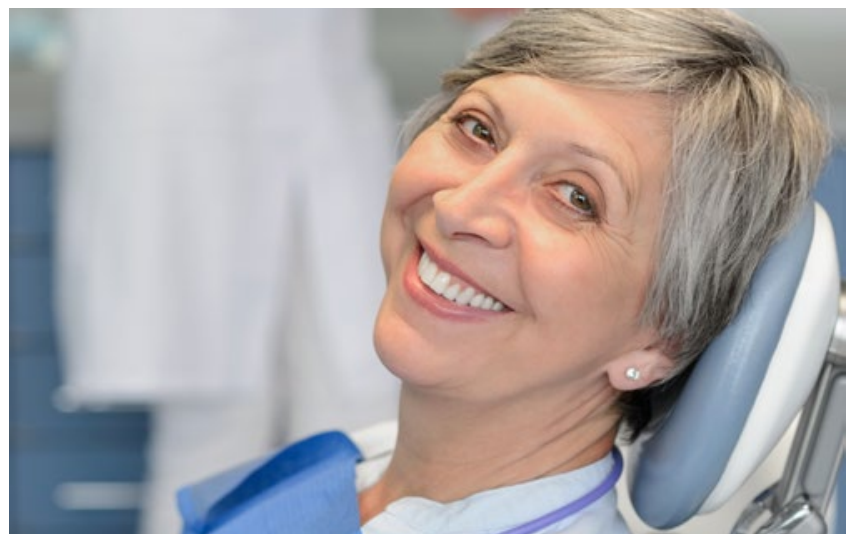

3-fach-Prophylaxe sinnvoll. Besonders empfehlenswert sind Mundspülungen, die die vier ätherischen Öle Thymol, Menthol, Methylsalicylat und Eukalyptol enthalten, wie z.B. Listerine. Das zusätzliche Spülen mit einer antibakteriellen Mundspülung hemmt nachweislich signifikant die Aktivität und Vermehrung der Plaquebakterien, insbesondere in den für Zahnbürste und Zahnseide schwer zugänglichen Bereichen des Mundraums, und verlangsamt zusätzlich die Neubildung des dentalen Biofilms. ${ }^{6,7}$

Weitere Informationen finden Sie auf www.listerineprofessional.de Quellen:

${ }^{1}$ Altersgruppe Jüngere Senioren: 65 - bis 74-Jährige

${ }^{2}$ Fünfte Deutsche Mundgesundheitsstudie (DMS V) - Kurzfassung, Institut der Deutschen Zahnärzte im Auftrag von Bundeszahnärztekammer und Kassenzahnärztlicher Bundesvereinigung. 6-8; 20-26.

${ }^{3}$ Overholser $C D$ et al. Comparative effects of 2 chemotherapeutic mouthrinses on the development of supragingival dental plaque and gingivitis. J Clin Periodontol 1990; 17: 575579.

${ }^{4}$ Das Prophylaxekonzept für die Zahnarztpraxis. Grundlagen. Band 1. Zahnärztlicher FachVerlag (2013); 90.

Dr. med. dent. Aneta Pecanov-Schröder (2013): Für jedes Alter die richtige Prophylaxe, Bonn. http://www.dzw.de/artikel/für-jedes-alter-die-richtige-prophylaxe.

${ }^{6}$ Fine DH, Markowitz K, Furgang D, Goldsmith D, Ricci-Nittel D, Charles CH, Peng P, Lynch $M C$. Effect of rinsing with an essential oil-containing mouthrinseon subgingival periodontopathogens. J Periodontol 2007; 78: 1936-1942.

${ }^{7}$ Fine $\mathrm{DH}$ et al.: Effect of an essential oil-containing antiseptic mouthrinse on plaque and salivary Streptococcus mutans levels.J Clin Periodontol 2000; 27(3): 157-61. 